\title{
Total Variation Based Image Restoration of Three Dimensional Microscopic Objects
}

\author{
Michael K. Ng \\ Computer Sciences Laboratory \\ The Australian National University, Australia
}

\begin{abstract}
The inverse problem involving the determination of a three-dimensional biological structure from images obtained by means of optical-sectioning microscopy is ill-posed. Regularization methods must often be used in order to obtain a reasonable solution. Recently, the Total Variation (TV) regularization, as proposed by Rudin, Osher and Fatemi [11], has become very popular for this purpose. An iterative algorithm is used for minimizing a TV-penalized least squares problems. We also employ transform based methods for solving large linear subproblems arising from TV-penalized least problems. Preliminary numerical results show that the method performs quite well.
\end{abstract}

\section{INTRODUCTION}

The quality of the recorded image is usually degraded by blurring and noise. Given the recorded image, the blurring function and the noise distribution, the image restoration problem is to find an approximation to the original image.

\subsection{Background}

Light microscopy is a powerful tool for the noninvasive examination of biological specimens, see [1]. Specimens are frequently labeled with fluorescent probes that are specific for defined molecular components within cells. Visualization of these fluorescent probes in three dimensions is critically important for understanding the three-dimensional (3-D) architectures of cells and cellular components. Computational opticalsectioning microscopy is one method for reconstructing three-dimensional (3-D) images of living biological structures from data acquired by using light microscopy. These data are obtained by first labeling the specimen with a dye that fluoresces when exposed to light. Then a series of twodimensional (2-D) is collected while the specimen is stepped through focus. Since photons are detected from anywhere in the specimen, each 2-D image contains information from both the current in-focus plane and the out-of-focus planes. The effect of the photons from out-of-focus regions can be described by modeling the microscope's optics and the detection process, see $[6,12]$.

Assume that the 3-D specimen $f(x, y, z)$ with some thickness $\tau$ of interest is transparent and emits light incoherently. The impulse response of the optical system of the microscope is obtained from the image of a point source and is called the incoherent point-spread function (PSF), $a(x, y, z)$. The data acquired in fluorescence imaging of a thick specimen by using optical sectioning, in which each image corresponds to a different focal-plane setting of the microscope. The data image $g(x, y, z)$ is given by

$$
\begin{aligned}
g(x, y, z)= & \int_{0}^{\tau} \int_{\Omega} a(x-s, y-t, z-\nu) f(s, t, \nu) d s d t d \nu \\
& +\eta(s, t, \nu) \text { or } g=\mathcal{A} f+\eta
\end{aligned}
$$

where $\eta(x, y, \nu)$ models the noise in the system, $\mathcal{A}$ is the convolution operator corresponding to the PSF $a(x, y, z)$. We note that light emanating from all sections in the object contributes to the image data formed for the focal plane at $z$. The set of data images locating along $z$-axis, is then the result of the 3 -D convolution of $f(x, y, z)$ with $a(x, y, z)$ over the dimensions $x, y, z$. In this paper, we need to simultaneously deconvolve and denoise the sequence of the recorded 2-D images during the reconstruction process.

\subsection{Total Variation Regularization}

In general, the problem $\mathcal{H} f=g$, with $\mathcal{H}$ a compact operator, ill-posed. It is not worth solving this equation for the data is assumed to be inexact, and the solution would be highly oscillatory. However, if we impose a certain regularity condition on the solution $f$, then the method becomes stable. We can consider the Tikhonov regularization and solve the following constrained problem:

$$
\min _{f} R(f) \text { subject to }\|\mathcal{H} f-g\|_{2}=\sigma,
$$

where $R(\cdot)$ is a certain functional which measures the irregularity of $f$ in a certain sense and $\sigma$ is the 
noise level. For instance, $R(f)=\|f\|_{2}^{2},\left\|\mathcal{D}_{k} f\right\|_{2}^{2}$, where $D$ is a $k$ th order differential operator. The algorithms for deblurring and noise removal have been mainly based on least squares. The output of these least squares based algorithms will be a continuous or smooth function, which cannot obviously be a good approximation to original image if it contains edges. To overcome this difficulty a technique based on the minimization of the Total Variation norm subject to some noise and blurring constraints is proposed by Rudin, Osher and Fatemi [11]. They proposed to use as regularization function the so-called Total Variation norm:

$$
\begin{aligned}
& T V(f)=\int_{\Omega}|\nabla f| d x d y= \\
& \int_{\Omega} \sqrt{\left(\frac{\partial f}{\partial x}\right)^{2}+\left(\frac{\partial f}{\partial y}\right)^{2}} d x d y .
\end{aligned}
$$

The solution to the (2) for $R(f)=T V(f)$ can have discontinuities, thus allowing us to recover the edges of the original image from the blurred and noisy data. Recently, Oman and Vogel [10] considered the following closely-related regularization problem:

$$
\min _{f} \frac{1}{2}\|\mathcal{H} f-g\|_{2}^{2}+\alpha \int_{\Omega} \sqrt{|\nabla f|^{2}+\beta} d x d y,
$$

where $\alpha$ and $\beta$ are positive parameters. The parameter $\alpha$ controls the tradeoff between goodness of fit to the data and the variability of the solution. At a stationary point of (3), its gradient vanishes, giving:

$$
\begin{gathered}
\mathcal{H}^{*}(\mathcal{H} f-g)-\alpha \nabla \cdot\left(\frac{\nabla f}{\sqrt{|\nabla f|^{2}+\beta}}\right)=0, \\
\text { on } \quad(x, y), \in \Omega \quad \text { with } \quad \frac{\partial f}{\partial n}=0, \quad(x, y) \in \partial \Omega .
\end{gathered}
$$

The gradient equation is a non-degenerate nonlinear second order elliptic partial differential equation. We note that the parameter $\beta$ added is used to remove the degeneracy of the diffusion equation.

Many numerical schemes have been devised in 2-D image restoration to obtain minimizer of the functional by solving the the gradient equation directly. For example, in [11], an explicit time marching scheme is used. In [10], Oman and Vogel introduced the fixed point iteration to solve the gradient equation (4). The advantage is that the fixed point method exhibits rapid linear convergence for a broad range of the parameters $\alpha$ and $\beta$.

In this paper, the minimization problem is extended to restore 3-D images of microscopic objects. The outline of this paper as follows. In the next section, we consider the Total Variation regularization problem of the 3-D microscopic objects. Computational speed is often of the utmost importance to the biological researcher who may evaluate dozens of specimens in an experiment before selecting one for continued study. In addition, researchers often examine living specimens in time-lapse analyses. In these applications, processing speed is critical for determining whether the specimen is behaving properly during the course of the experiment. We also study a fast algorithm to solve the 3-D Total Variation regularization problem. Finally, some preliminary numerical results are reported to show the effectiveness of our method.

\section{3-D TOTAL VARIATIONAL REGU- LARIZATION METHOD}

The problem of interest is that of determining the object's intensity $f(x, y, z)$ in terms of data images defined in (1). We follow the approach in [10] and consider the following unconstrained regularization problem:

$$
\begin{gathered}
\min _{f} \frac{1}{2}\|\mathcal{A} f-g\|_{2}^{2}+ \\
\alpha \int_{\Omega} \sqrt{\left(\frac{\partial f}{\partial x}\right)^{2}+\left(\frac{\partial f}{\partial y}\right)^{2}+\left(\frac{\partial f}{\partial z}\right)^{2}+\beta} d x d y d z .
\end{gathered}
$$

At a minimizer, we need to solve the nonlinear elliptic equation on $\Omega \times[0, \tau]$ :

$$
\begin{gathered}
G(f) \equiv \mathcal{A}^{*}(\mathcal{A} f-g)- \\
\alpha \nabla \cdot\left(\frac{\nabla f}{\sqrt{\left(\frac{\partial f}{\partial x}\right)^{2}+\left(\frac{\partial f}{\partial y}\right)^{2}+\left(\frac{\partial f}{\partial z}\right)^{2}+\beta}}\right)=0 \\
\text { with } \frac{\partial f}{\partial n}=0, \quad(x, y, z) \in \partial \Omega \times[0, \tau] .
\end{gathered}
$$

The fixed point iteration is also employed to solve the gradient equation (6).

\subsection{Discrete Equations}

In practice, the data acquired in fluorescence imaging of a thin specimen by using optical sectioning consist of a set of $m 2-\mathrm{D} n \times n$ images. The system of linear equations obtained from (1) for $k=1, \cdots, m$, where $m$ is the number of data images, can be written in a matrix-vector form by using a discrete space-invariant model. Let $g$ be a $m n^{2}$ stacked vector that is obtained from stacking the rows of $m, n \times n$ data images, $f$ the stacked version of the original object sections, and $\eta$ the noise vector. A block Toeplitz 
with block-Toeplitz-Toeplitz-block matrix $A$ can be constructed such that

$$
g=A f+\eta,
$$

where

$$
A=\left[\begin{array}{cccc}
A_{0} & A_{-1} & \cdots & A_{1-m} \\
A_{1} & A_{0} & & A_{m-2} \\
\vdots & & \ddots & \vdots \\
A_{m-1} & A_{m-2} & \cdots & A_{0}
\end{array}\right],
$$

where the blocks $A_{k}$ are themselves blockToeplitz-Toeplitz-block matrices of order $n^{2}$. Each $A_{k}$ is an $n^{2}$-by- $n^{2}$ matrix of the form given by (7) with each block is a Toeplitz matrix of order $n$.

On the other hand, by applying the usual 7point centered differencing scheme with $n$ internal grid nodes in $x$ and $y$ coordinate directions and $m$ internal grid nodes in $z$ coordinate directions, the resulting discretization matrix $L_{\beta}(f)$ will be an $m n^{2}$-by- $m n^{2}$ symmetric matrix of the form

$$
\left[\begin{array}{ccccc}
D_{1}(f) & E_{2}(f) & & & \\
E_{2}(f) & D_{2}(f) & E_{3}(f) & & \\
& \ddots & \ddots & \ddots & \\
& & \ddots & \ddots & E_{m}(f) \\
& & & E_{m}(f) & D_{m}(f)
\end{array}\right] .
$$

with $E_{i}$ and $D_{i}$ being $n^{2}$-by- $n^{2}$ diagonal matrices and $n^{2}$-by- $n^{2}$ tridiagonal block matrices respectively. Therefore, the fixed point iteration can be expressed as

$$
\left[A^{*} A+\alpha L_{\beta}\left(f_{k}\right)\right] f_{k+1}=A^{*} g, \quad k=0,1, \cdots .
$$

Noting from (10), we now need to solve a linear system with the coefficient matrix involving the convolution operator and elliptic operator for each fixed point iteration.

\subsection{Preconditioned Conjugate Gradient Method}

In this subsection, we apply the preconditioned conjugate gradient (PCG) method to solve (10) and we concentrate on finding a good preconditioner for (10). The version of the PCG algorithm we use is given in [8] in generic form, and can be stated as follows for our application:

PCG Algorithm:. Let $y_{0}$ be an approximation for solving $n-b y$-n linear system $K y=z$ and $P$ be the preconditioner for the matrix $K$. The algorithm computes the solution to a fixed accuracy $\epsilon$. Here $\phi_{j}$ and $\rho_{j}$ are scalars and $q_{j}, r_{j}$ and $p_{j}$ are $n$-vectors.
- $r_{0}=z-K y_{0}$

- $p_{0}=0$

- $\phi_{0}=1$

- $\tau_{0}=\left\|r_{0}\right\|_{2}$

- For $j=1,2, \ldots$ until $\tau_{j-1} / \tau_{0}<\epsilon$

$$
\begin{aligned}
& q_{j}=P^{-1} r_{j-1} \\
& \phi_{j}=q_{j}^{*} r_{j-1} / \phi_{j-1} \\
& p_{j}=q_{j}+\phi_{j} p_{j-1} \\
& \rho_{j}=q_{j}^{*} r_{j-1} / p_{j}^{*} K p_{j} \\
& y_{j}=y_{j-1}+\rho_{j} p_{j} \\
& r_{j}=r_{j-1}+\rho_{j} K p_{j} \\
& \tau_{j}=\left\|r_{j}\right\|_{2}
\end{aligned}
$$

Given a matrix $K$, there are two criteria for choosing a preconditioner for $K$, see Golub and Van Loan [8]. First, a preconditioner $P$ should be a "good" approximation to $K$. Secondly, it must be easily invertible. Recall that the coefficient matrix in (10) corresponds to the sum of a convolution operator and an elliptic operator. There are many "good" preconditioners for the individual parts. For example, for the elliptic part, we have the MINV-type preconditioners [5]. For the convolution part, we have transform based preconditioners [3]. In [2], Chan et al. investigated using transform based preconditioners which is easily invertible for 2-D Total Variation image restoration problems. Next we extend their approach to construct a preconditioner for the system $(10)$.

\subsection{Transform Based Preconditioning}

The concept of optimal transform approximation was first introduced by T. Chan [4]. Since preconditioners can be viewed as approximations to the given matrix $K$, it is reasonable to consider preconditioners which minimize $\|Q-K\|$ over all $Q$ belonging to some class of matrices and for some matrix norm $\|\cdot\|$. T. Chan [4] proposed the optimal circulant preconditioner that is the minimizer of the Frobenius norm $\|Q-K\|_{F}$ over the class of all circulant matrices $Q$. It has been proved that these preconditioners is very effective for solving convolution systems with the PCG method, see [3]. We note that the boundary condition of the nonlinear elliptic equation (6) is Neumann boundary condition. Therefore, Chan et al. [2] used the optimal cosine transform approximation to construct preconditioner for the system (10). Their theoretical and numerical results show that the performance of the optimal cosine transform based preconditioner perform very well

Let us denote $C_{n}$ to be the $n$-by- $n$ discrete cosine transform matrix. If $\delta_{i j}$ is the Kronecker delta, then the $(i, j)$ th entry of $C_{n}$ is given by

$\sqrt{\frac{2-\delta_{i 1}}{n} \cos \left(\frac{(i-1)(2 j-1) \pi}{2 n}\right)}, \quad 1 \leq i, j \leq n$, 
see Jain [9]. For our 3-D Total Variation image restoration problem, we consider the optimal cosine transform approximations $P_{1}$ and $P_{2}$ for the discretization matrices $A$ (convolution part) and $L_{\beta}\left(f_{k}\right)$ (elliptic part) respectively. More precisely, $P_{1}$ and $P_{2}$ are the minimizers of

$$
\|Q-A\|_{F} \text { and }\left\|Q-L_{\beta}\left(f_{k}\right)\right\|_{F}
$$

respectively over all matrices $Q$ that can be diagonalized by the matrix $C_{m} \otimes C_{n} \otimes C_{n}$. Here $\otimes$ is the Kronecker tensor product. The minimizers can be viewed as the approximations of $A$ and $L_{\beta}\left(f_{k}\right)$ along $x, y$ and $z$ directions, see [3] for details. We remark $P_{1}$ and $P_{2}$ can be computed in $O\left(m n^{2} \log n+n^{2} m \log m\right)$ operations using fast cosine transform. Hence the preconditioner $P$ for $A^{*} A+L_{\beta}\left(f_{k}\right)$ in $(10)$ can be defined as

$$
P=P_{1}^{*} P_{1}+\alpha P_{2}
$$

Since $P_{1}$ and $P_{2}$ can be diagonalized by the matrix $C_{m} \otimes C_{n} \otimes C_{n}$, the preconditioner $P$ is also and hence the cost of constructing the preconditioner $P$ is in $O\left(m n^{2} \log n+n^{2} m \log m\right)$ operations.

The cost of one PCG iteration is bounded by the cost of the matrix vector multiplications $\left(A^{*} A+L_{\beta}\left(f_{k}\right)\right) v$ and the cost of solving the system $P y=z$. The matrix-vector multiplications $A^{*} A v$ and $\left.L_{\beta}\left(f_{k}\right)\right) v$ can be computed in $O\left(m n^{2} \log n+n^{2} m \log m\right)$ operations using fast cosine transforms and $O\left(m n^{2}\right)$ operations using the band structure of $\left.L_{\beta}\left(f_{k}\right)\right)$ respectively. The system $P y=z$ can be solved in $O\left(m n^{2} \log n+n^{2} m \log m\right)$ operations by exploiting the fast cosine transforms again. Therefore the total cost of each PCG iteration is bounded by of $O\left(m n^{2} \log n+n^{2} m \log m\right)$ operations.

\section{NUMERICAL RESULTS}

To see the performance of the Total Variation based image restoration method, computer simulations are performed. The $3-\mathrm{D}$ phantom uses in the simulations consists of six disjoint geometrical volumes in the $x, y$ and $z$ space, as shown in Figure 1. This phantom is chosen because it varies in all three dimensions and has sharp edges. In the simulations, $1664 \times 64$ images of the simulated data are used. Figures 2 and 3 shows the $x-y$ section and $x-z$ section images of the phantom specimen. The images shown in Figures 4 and 5 are the $x-y$ and $x-z$ sections through the simulated microscopic data that are obtained from the 3$\mathrm{D}$ discrete convolution of the phantom, $a(x, y, z)$, with $f(x, y, z)$ in Figure 1 and contain additive white Gaussian noise. The SNR for each section image is 0.02 . For this simulation study, a family of 3-D Gaussian functions is used as an approximation of the microscope's PSF. Some of these 2D Gaussian functions are shown in Figure 6. We perform the fixed point iterations until the gradient in (6) satisfies $\left\|G\left(f_{k}\right)\right\|_{2} /\left\|G\left(f_{0}\right)\right\|_{2} \leq 10^{-2}$. We apply the preconditioned conjugate gradient method to solve the linear system (10) with cosine transform based preconditioner discussed in $\$ 2.3$. The stopping criterion of the preconditioned conjugate gradient method is $\left\|r_{k}\right\|_{2} /\left\|r_{0}\right\|_{2}<10^{-3}$, where $r_{k}$ is the residual vector of the linear system (10) at the $k$-th PCG iteration. Tables 1 shows the total numbers of $C G$ iterations required for convergence with and without using the preconditioner. In Figures 7 and 8, we present TV-based image restorations described in $\S 2$. For the sake of comparison we have used the $L_{2}$-norm $\left(=\int|f|^{2}\right)$ as regularization functional to restore the blurred and noisy image. The result is shown in Figures 9 and 10 . In summary, the preliminary experiment suggests that the total variation based regularization method with the PCG algorithm may be an efficient and effective method for 3-D microscopic imaging problems.

\section{REFERENCES}

1. D. Agard, Y. Hiraoka, P. Shaw and J. Sedat, Fluorescence Microscopy in Three Dimensions, Methods Cell Biol., 30 (1989), pp. $353-377$.

2. R. Chan, T. Chan and C. Wong, Cosine Transform Based Preconditioners for Total Variation Minimization Problems in Image Processing, Proceedings of The Second IMACS International Symposium on Iterative Methods in Linear Algebra, S. Margenov and P. Vassilevski, eds., Bulgaria, 1995.

3. R. Chan and M. Ng, Conjugate Gradient Methods for Toeplitz Systems, to appear SIAM Review (1996).

4. T. Chan, An Optimal Circulant Preconditioner for Toeplitz Systems, SIAM J. Sci. Stat. Comput., 9 (1988), pp. 766-771.

5. P. Concus, G. Golub and G. Meurant, Block Preconditioning for the Conjugate Gradient Method, SIAM J. Sci. Statist. Comput., 6 (1985), pp. 220-252.

6. A. Erhardt, G. Zinser, D. Komitiwski and J. Bille, Reconstruction $3 D$ Light Microscopic Images by Digital Image Processing, Appl. Opt., 24 (1985), pp. 194-200. 


\begin{tabular}{|c||c|}
\hline Without using preconditioner & 4505 \\
\hline with using preconditioner & 1455 \\
\hline
\end{tabular}

Table 1: Number of CG iterations.
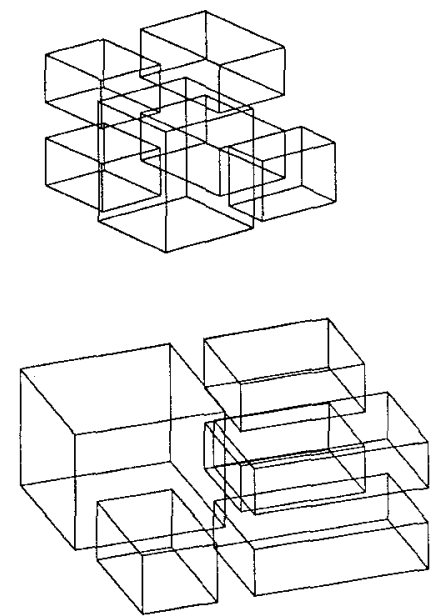

Figure 1: Different views of the phantom consisting of seven structures.

7. F. Gibson and F. Lanni, Diffraction by a Circular Aperture as a Model for Threedimensional Optical Microscopy, J. Opt. Soc. Am., A6 (1989), pp. 1357-1367.

8. G. Golub and Van Loan, Matrix Computations, Johns Hopkins Press, Baltimore, Second Edition (1989).

9. A. Jain, Fundamentals of Digital Image Processing, Prentice Hall, London, 1989.

10. M. Oman and C. Vogel, Iterative Methods for Total Variation Denoising, to appear SIAM J. Sci. Comput. (1996).

11. L. Rudin, S. Osher and E. Fatemi, Nonlinear Total Variation Based Noise Removal Algorithms, Physica D., 60 (1992), pp. 259-268.

12. M. Weinstein and K. Castleman, Reconstructing $3 D$ Specimens from $2 D$ Section Images, Quantitative Imagery in the Biomedical Sciences I, R. Herron,ed., Proc. Soc. Photo-Opt. Instrum. Eng., 26 (1971), pp. 131-138.

5. ACKNOWLEDGMENTS: Research by $\mathrm{M}$. $\mathrm{Ng}$ was supported by the Cooperative Research Centre for Advanced Computational Systems.

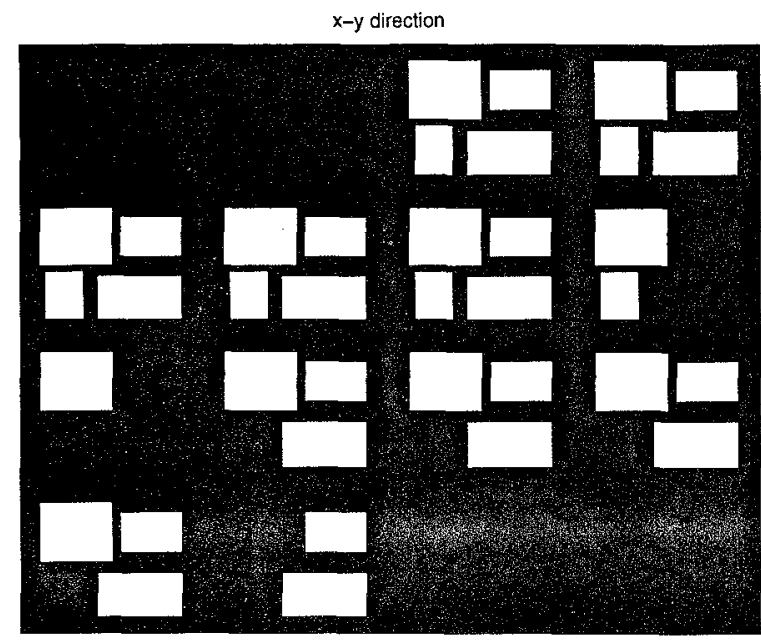

Figure 2: $x-y$ section images of the intensity of the phantom.

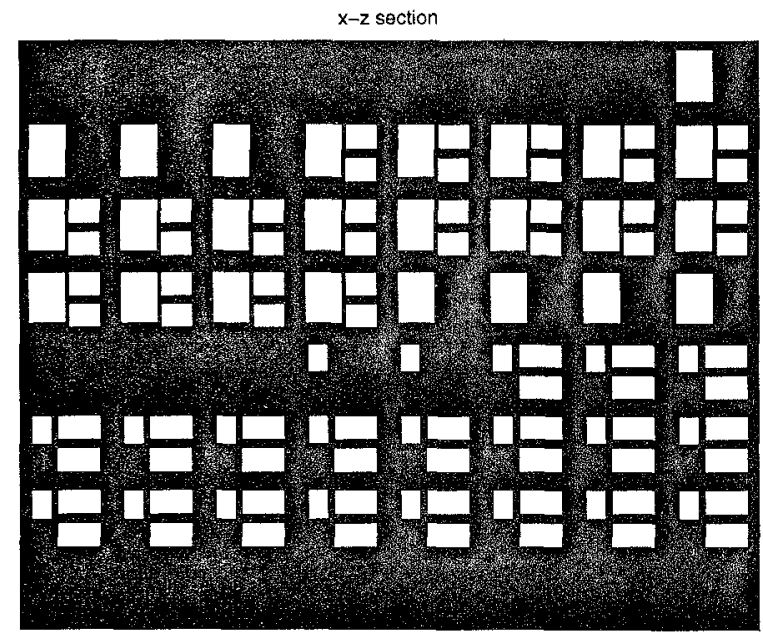

Figure 3: $x-z$ section images of the intensity of the phantom.

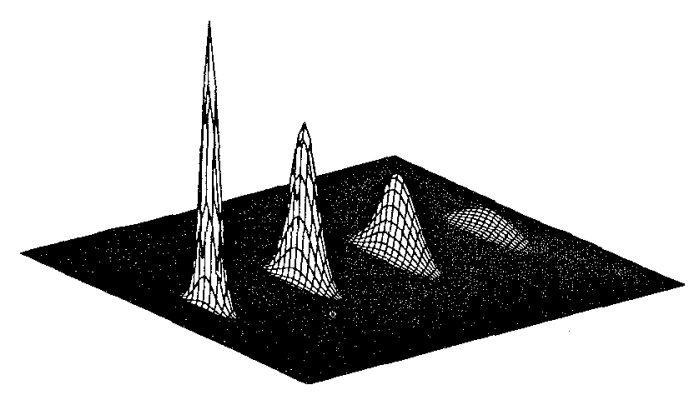

Figure 4: Different 2-D Gaussian functions. 


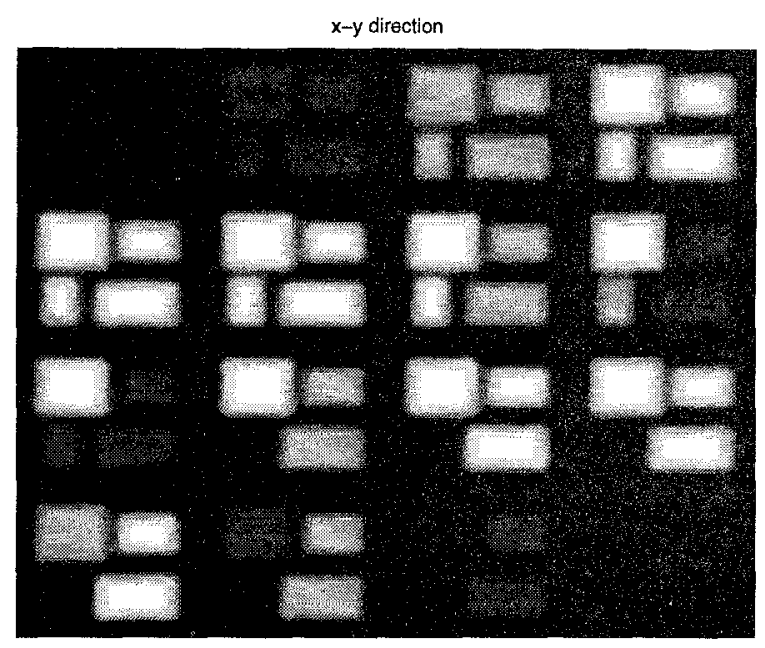

Figure 5: $x-y$ section images of the intensity of the blurred and noisy phantom.

$\mathbf{x}-\mathbf{2}$ section

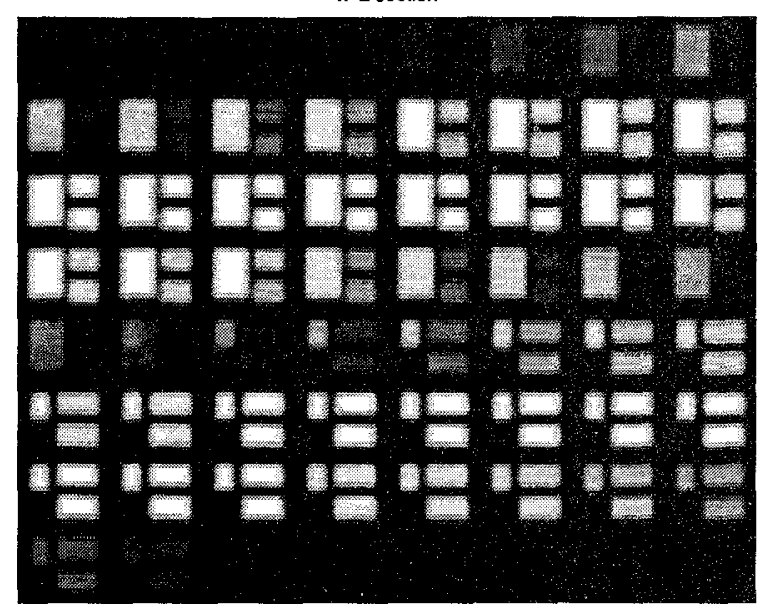

Figure 6: $x-z$ section images of the intensity of the blurred and noisy phantom.

\section{$x-y$ direction}

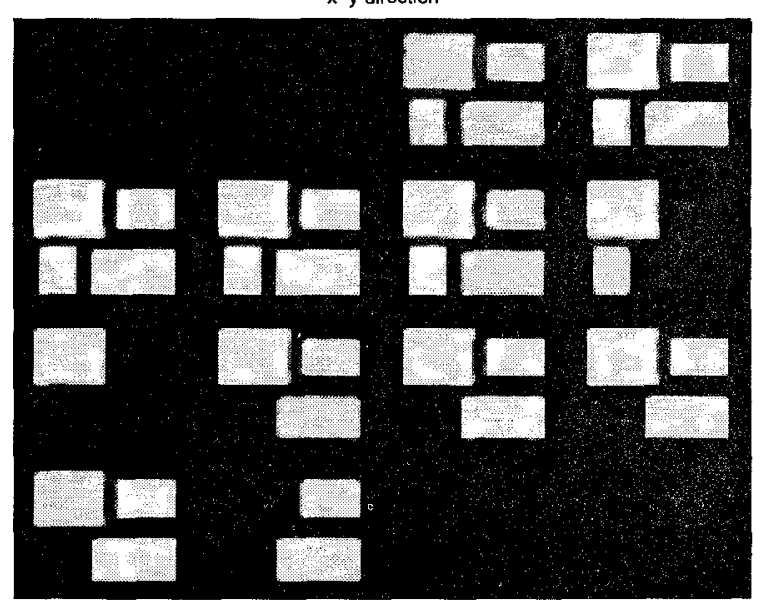

Figure 7: $x-y$ section images of the intensity of the restored phantom using TV regularization.

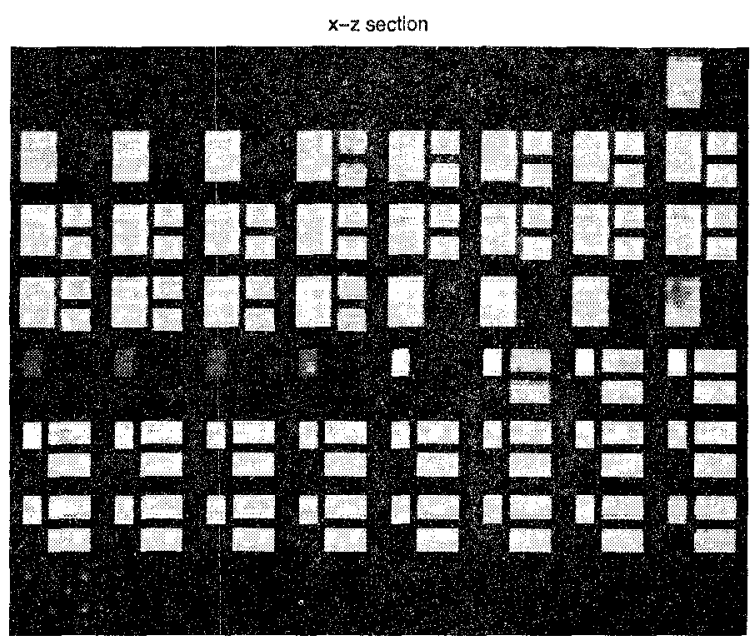

Figure 8: $x-z$ section images of the intensity of the restored phantom using TV regularization.

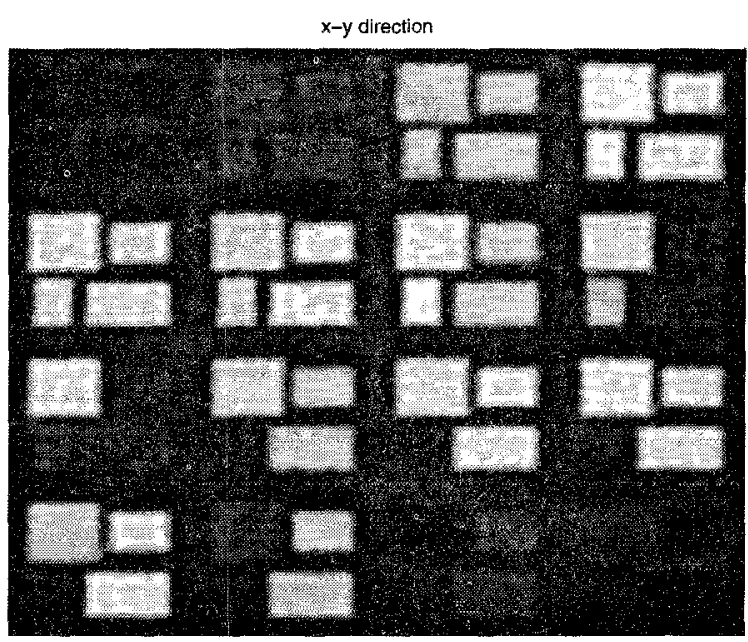

Figure 9: $x-y$ section images of the intensity of the restored phantom using $L_{2}$ regularization.

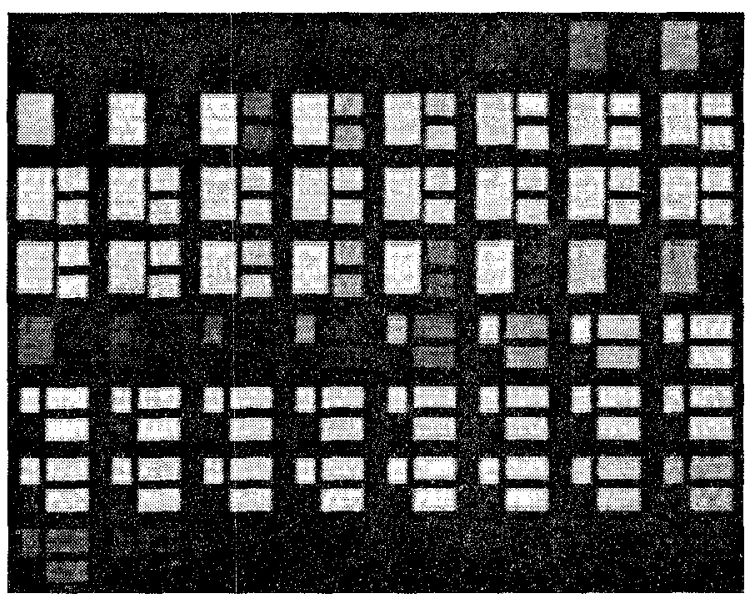

Figure 10: $x-z$ section images of the intensity of the restored phantom using $L_{2}$ regularization. 